Received: 22.10 .2021

Revised: 16.12 .2021

Accepted: 24.12 .2021

DOI: $10.17804 / 2410-9908.2021 .6 .037-044$

\title{
STUDYING THE PROCESS OF PULSED LOADING OF A WATER-FILLED CYLINDRICAL CONTAINER
}

\author{
I. I. Valov \\ Chelyabinsk State University, Miass Branch, \\ 1 Kerchenskaya St., Miass, Chelyabinsk Region, 456313, Russian Federation \\ South Ural Federal Scientific Center of Mineralogy and Geoecology, \\ Ural Branch of the Russian Academy of Sciences, Ilmeny Reserve, \\ Miass, Chelyabinsk Region, 456317, Russian Federation \\ (D) https://orcid.org/0000-0001-9790-2788 @ ofpat@mail.ru \\ Corresponding author. E-mail: vii_1982@mail.ru \\ Address for correspondence: Pr. Oktyabrya, 16, Miass, Chelyabinsk Region, 456318, Russian Federation \\ Tel.: +7 (3513) 2555 43; (3513) 591551
}

The paper solves the problem of determining the pressure field in a liquid during experimental testing of the motion of objects under water. A calculation method is given for determining the pressure field on the walls of a cylindrical container during the intensive development of a spherical gas cavity in a liquid. The presence of solid container walls is taken into account by placing fictitious sources on them with an intensity that ensures the fulfillment of the nonleakage condition. The law of development of a gas bubble, similar to the Rayleigh equation, is derived; the mass of gas in the bubble is determined in accordance with the Saint-Venant equation. The pressure at the container point of interest to the researcher is calculated with the use of the Cauchy-Lagrange integral. The constructed method makes it possible to estimate the loads on the experimental stand in the event of an emergency situation.

Keywords: hydrodynamic experiment, pressure field in a liquid.

\section{References}

1. Kochin N.E., Kibel I.A., Roze N.V. Teoreticheskaya gidromekhanika [Theoretical Hydromechanics]. Moscow, Fizmatgiz Publ., 1964, vol. 1, 584 p. (In Russian).

2. Milne-Thompson L. M. Teoreticheskaya gidromekhanika [Theoretical Hydrodynamics]. Moscow, Mir Publ., 1964, 660 p. (In Russian).

3. Sedov L.I. Mekhanika sploshnoy sredy [Continuum Mechanics]. Moscow, Nauka Publ., 1970, vol. 2, 568 p. (In Russian).

4. Chizhiumov S.D. Osnovy gidromekhaniki [Fundamentals of Hydrodynamics: a tutorial]. Komsomolsk-on-Amur, KnAGTU Publ., 2007, 106 p. (In Russian).

5. Degtyar V.G., Pegov V.I. Gidromekhanika ballisticheskikh raket podvodnykh lodok [Submarine Ballistic Missile Hydrodynamics]. Miass, KB im. Akad. V.P. Makeeva Publ., 2004, 256 p. (In Russian).

6. Degtyar V.G., Pegov V.I., Merkulov E.S. Numerical simulation of the evolution of the cavity boundary during torpedo launch. Vestnik YuURGU. Seriya Matematicheskoe Modelirovanie $i$ Progrmmirovanie, 2013, 6 (1), pp. 5-12. (In Russian).

7. Spevak L.F., Nefedova O.A. Parallelizing the Solution of the Nonlinear Heat Conduction Problem with the Application of the Opencl Library. Diagnostics, Resource and Mechanics of materials and structures, 2016, iss. 6, pp. 80-91. DOI: 10.17804/2410-9908.2016.6.080-091. Available at: http://dream-journal.org/issues/2016-6/2016-6_113.html (accessed: 12.12.2018). 
8. Kazakov A.L., Spevak L.F., Nefedova O.A. Simultenious Application of the Boundary Element Method and the Power Series Method for Solving a Two-Dimensional Problem of Heat Wave Motion. Diagnostics, Resource and Mechanics of materials and structures, 2017, iss. 6, pp. 6-15. DOI: 10.17804/2410-9908.2017.6.006-015. Available at: http://dream-journal.org/issues/20176/2017-6_151.html (accessed: 12.12.2018).

9. Pegov V.I., Moshkin I.Yu. Applying the Method of Plane Sections for Evaluating the Parameters of Flight Vehicles under Multiphase Flow. Diagnostics, Resource and Mechanics of materials and structures, 2020, iss. 4, pp. 48-61. DOI: 10.17804/2410-9908.2020.4.048-061. Available at: http://dream-journal.org/issues/2020-4/2020-4_299.html (accessed: 23.12.2021).

10. Pegov V.I., Moshkin I.Yu. Applying An Updated Method of Markers to Defining Transient Force Impact under Multiphase Flowing. Diagnostics, Resource and Mechanics of materials and structures, 2020, iss. 5, pp. 45-57. DOI: 10.17804/2410-9908.2020.5.045-057. Available at: http://dream-journal.org/issues/2020-5/2020-5_300.html (accessed: 23.12.2021). 
Подана в журнал: 22.10 .2021

УДК 532.5

DOI: $10.17804 / 2410-9908.2021 .6 .037-044$

\title{
ИССЛЕДОВАНИЕ ПРОЦЕССА ИМПУЛЬСНОГО НАГРУЖЕНИЯ ЦИЛИНДРИЧЕСКОЙ ЕМКОСТИ, ЗАПОЛНЕННОЙ ВОДОЙ
}

\author{
И. И. Валов \\ Миасский филиал ФГБОУ ВО «ЧелГУ», \\ 1, ул. Керченская, Челябинская область, г. Миасс, 456313, Российская Федерация \\ Федеральное государственное бюджетное учреждение науки Южно-Уральский федеральный научный иентр \\ минералогии и геоэкологии Уральского отделения Российской академии наук, \\ тер. Ильменский заповедник, г. Миасс, Челябинская область, 456317, Российская Федерация \\ (DD https://orcid.org/0000-0001-9790-2788@ ofpat@mail.ru \\ Электронный адрес: vii_1982@mail.ru \\ Адрес для переписки: пр. Октября, 16, г. Миасс, Челябинская область, 456318, Российская Федерация \\ Тел.: (3513) 25-55-43; (3513) 59-15-51
}

Статья посвящена решению задачи определения поля давлений в жидкости при экспериментальной отработке движения объектов под водой. Приводится методика расчетов по определению поля давлений на стенки цилиндрической емкости при интенсивном развитии в жидкости сферической газовой полости. Наличие твердых стенок емкости учитывается путем размещения на них фиктивных источников с интенсивностью, обеспечивающей выполнение условия непротекания. Выведен закон развития газового пузыря, аналогичный уравнению Рэлея, масса газа в пузыре определена в соответствии с уравнением Сен-Венана. Давление в интересующей исследователя точке емкости находится с использованием интеграла Коши-Лагранжа. Построенная методика дает возможность оценить нагрузки на экспериментальный стенд в случае возникновения нештатной ситуации.

Ключевые слова: гидродинамический эксперимент, поле давлений в жидкости.

\section{1. Введение}

При исследовании вопросов движения подводных аппаратов на экспериментальных установках всегда существует риск возникновения нештатных ситуаций, связанных с отказом материальной части. Одним из возможных случаев является разрушение энергоузла, приводящего в движение модель подводного аппарата, в результате чего за короткий отрезок времени в ограниченную область внутри жидкости высвобождается достаточно большая масса газа, что приводит к значительному повышению давления внутри экспериментальной установки.

Поиск точного решения большинства практических задач гидродинамики, описываемых системами нелинейных дифференциальных уравнений (как правило, в форме задачи Коши или уравнений Навье-Стокса), сопряжен с большими трудностями, поэтому специалисты часто обращаются к численным методам решения. При этом для практикаэкспериментатора, сталкивающегося с необходимостью в сжатые сроки спланировать и провести серию экспериментов, актуальна задача описания исследуемого процесса инженерной математической моделью, допускающей меньшую точность решения по сравнению с решением, полученным с помощью более точных сеточных методов, но позволяющей предсказать характер исследуемого процесса, диапазоны и периоды нарастания измеряемых величин, а также за короткое время без применения кластерных технологий просчитать несколько вариантов с различными условиями эксперимента. В рассматриваемом случае исследователю желательно владеть методикой оценки возникающих давле- 
ний, чтобы обоснованно выбрать параметры заряда энергоузла, не приводящие к разрушению модельной установки, а также обоснованно выбрать запас прочности при проектировании новых экспериментальных стендов.

Инженерная модель развития газовой каверны, формируемой при выходе объекта из расположенной под водой стартовой установки, построена в монографии [5], а в работе [6] рассматривается алгоритм решения задачи методом конечных элементов и приводятся результаты расчета. Построению и практической реализации методов решения задач механики сплошных сред, описывающих высокоинтенсивные процессы, сопровождающиеся значительным изменением одной из величин за малые промежутки времени, посвящены исследования $[7,8]$. Полученные результаты дают возможность существенно повысить точность решения и сократить необходимое для расчета время. Вопросам взаимодействия подводных и летательных аппаратов с многофазными потоками и возникающим при этом нагрузкам на конструкцию посвящены исследования $[9,10]$, при этом задача решается методами численного имитационного моделирования на вычислительном кластере.

В настоящей работе предложена инженерная математическая модель расчета силового нагружения вертикально расположенной цилиндрической емкости (радиус емкости $R$, заполненной водой на высоту $L$ ) в случае интенсивного развития в ней единичной газовой полости, пригодная для проведения в короткий срок серии расчетов на обычном персональном компьютере.

\section{2. Постановка задачи и методы решения}

Будем решать задачу при следующих допущениях:

- жидкость невязкая и несжимаемая, ее движение потенциальное. В начальный момент времени жидкость находится в состоянии покоя;

- газовая полость развивается, сохраняя сферическую форму, и движется строго вверх с известной скоростью $U$;

- изменением уровня жидкости в экспериментальной установке пренебрегаем.

Газовую полость будем рассматривать как особенность - источник. Обильность такого источника $Q$ определяется объемом жидкости, переносимым через контрольную поверхность (пусть это сфера известного радиуса) в единицу времени. В силу сделанного предположения о невязкой и несжимаемой жидкости $Q=\frac{d V}{d t}$ и для потенциала источника также имеем: $\Phi=\frac{1}{4 \pi \rho} \frac{d V}{d t}$. Здесь $\rho$ - расстояние от центра особенности до точки, значение потенциала в которой нас интересует, $V$ - объем полости [2].

Условие непротекания на границе области $\frac{\partial \Phi}{\partial n}=0$ удовлетворим следующим образом:

- изменение радиуса газовой сферы ( $a$ - радиус газовой полости; $\&$ - скорость его изменения) учтем, разместив на каждом участке цилиндрической поверхности площадью $d S$ (нижнее основание и боковые стенки) фиктивный элементарный источник с обильностью [4]:

$$
d Q=\frac{d S \cdot\left(h, \frac{\rho_{1}}{\rho_{1}}\right)}{4 \pi \rho_{1}^{2}} \frac{d V}{d t}=\frac{a^{2} \& d S \cdot\left(h, \frac{\rho}{\rho_{1}}\right)}{\rho_{1}^{2}}
$$


- движение сферы вверх со скоростью $U$ учтем, разместив симметрично плоскости нижнего основания цилиндрической области фиктивную сферу, движущуюся со скоростью $U$ в противоположном направлении (рис. 1).

Тогда итоговый потенциал течения в рассматриваемой области, учитывающий влияние источника - газовой полости, фиктивных источников на стенках экспериментальной установки и дополнительной фиктивной сферы, запишется как $[2,4]$ :

$$
\begin{aligned}
& \Phi= \frac{a^{2} \&}{\rho_{3}}+\int_{0}^{R} \int_{0}^{2 \pi}\left[\frac{1}{4 \pi\left(\rho_{1}+\rho_{2}\right)} \frac{1}{4 \pi \rho_{1}^{2}}\left(\rho, \frac{\rho_{1}}{\left|\rho_{1}\right|}\right) \frac{d V}{d t}\right] r d r d \varphi+ \\
&+\int_{0}^{L} \int_{0}^{2 \pi}\left[\frac{1}{4 \pi\left(\rho_{1}+\rho_{2}\right)} \frac{1}{4 \pi \rho_{1}^{2}}\left(\rho, \frac{\rho_{1}}{\left|\rho_{1}\right|}\right) \frac{d V}{d t}\right] R d z d \varphi+\frac{1}{2} U a^{3}\left[\frac{\cos \theta_{1}}{\rho_{3}}+\frac{\cos \theta_{2}}{\rho_{4}}\right]= \\
&=\frac{a^{2} \&}{\rho_{3}}+\int_{0}^{R} \int_{0}^{2 \pi}\left[\frac{a^{2} \&}{4 \pi\left(\rho_{1}+\rho_{2}\right) \rho_{1}^{2}}\left(\rho, \frac{\rho_{1}}{\left|\rho_{1}\right|}\right)\right] r d r d \varphi+ \\
&+\int_{0}^{L} \int_{0}^{2 \pi}\left[\frac{a^{2} \&}{4 \pi\left(\rho_{1}+\rho_{2}\right) \rho_{1}^{2}}\left(h, \frac{\rho_{1}}{\left|\rho_{1}\right|}\right)\right] R d z d \varphi+\frac{1}{2} U a^{3}\left[\frac{\cos \theta_{1}}{\rho_{3}}+\frac{\cos \theta_{2}}{\rho_{4}}\right] .
\end{aligned}
$$

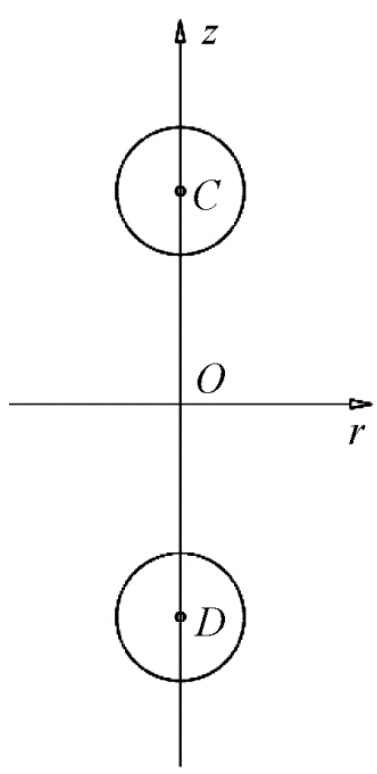

Рис. 1. Реальная и фиктивная сферы

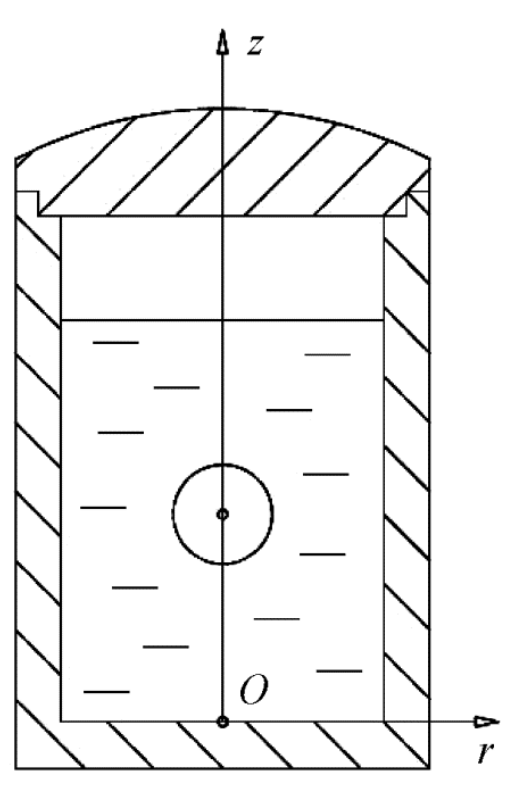

Рис. 2. Экспериментальная установка в разделе

Перейдем к цилиндрической системе координат, начало которой расположим в центре нижнего основания цилиндрической области, в которой ищем решение задачи, ось $z$ направим вверх по оси цилиндра (рис. 2).

Обозначим: $C\left(r_{C}, \varphi_{C}, z_{C}\right)$ - координаты центра газовой полости; $A\left(r_{A}, \varphi_{A}, z_{A}\right)-$ координаты точки, значение давления в которой нас интересует. Тогда:

$$
\rho_{1}=\sqrt{r^{2}+r_{C}^{2}-2 r r_{C} \cdot \cos \left(\varphi-\varphi_{C}\right)+\left(z-z_{C}\right)^{2}}-\text { расстояние от центра газовой полости }
$$

до точки с координатами $(r, \varphi, z)$, расположенной на границе области; 


$$
\rho_{2}=\sqrt{r^{2}+r_{A}^{2}-2 r r_{A} \cdot \cos \left(\varphi-\varphi_{A}\right)+\left(z-z_{A}\right)^{2}} \text { - расстояние от точки с координатами }
$$

$(r, \varphi, z)$, расположенной на границе области, до точки, значение потенциала (и давления) в которой нас интересует;

$$
\rho_{3}=\sqrt{r_{A}^{2}+r_{C}^{2}-2 r_{A} r_{C} \cdot \cos \left(\varphi_{A}-\varphi_{C}\right)+\left(z_{A}-z_{C}\right)^{2}}-\text { расстояние от центра сферической }
$$
полости до точки $A\left(r_{A}, \varphi_{A}, z_{A}\right)$;

$$
\rho_{4}=\sqrt{r_{A}^{2}+r_{D}^{2}-2 r_{A} r_{D} \cdot \cos \left(\varphi_{A}-\varphi_{D}\right)+\left(z_{A}-z_{D}\right)^{2}}=\sqrt{r_{A}^{2}+r_{C}^{2}-2 r_{A} r_{C} \cdot \cos \left(\varphi_{A}-\varphi_{C}\right)+\left(z_{A}+z_{C}\right)^{2}}-
$$

расстояние от центра фиктивной сферической полости до точки $A\left(r_{A}, \varphi_{A}, z_{A}\right)$.

Тогда давление в произвольной точке области $A\left(r_{A}, \varphi_{A}, z_{A}\right)$ может быть найдено из интеграла Коши-Лагранжа [3]:

$$
\frac{P-P_{c m}}{\rho}=\frac{\partial \Phi}{\partial t}-\frac{1}{2}\left(\frac{\partial \Phi}{\partial r}\right)^{2},
$$

где $P_{c m}$ - гидростатическое давление на глубине расположения газовой полости; $\rho$ - плотность жидкости.

Рассмотрим случай, когда расположенный на оси $z$ центр газовой полости (имеет координаты $C\left(0,0, z_{C}\right)$ ) (рис. 2). В этом случае формулы упрощаются. Проводя дифференцирование, получаем:

$$
\begin{gathered}
\frac{\partial \Phi}{\partial t}=\left(2 a \&+a^{2} d \int_{0}^{L} \int_{0}^{2 \pi} \frac{R^{2}}{\left(\rho_{1}+\rho_{2}\right) \rho_{1}^{3}} d z d \varphi+\int_{0}^{R} \int_{0}^{2 \pi} \frac{r \cdot z_{C}}{\left(\rho_{1}+\rho_{2}\right) \rho_{1}^{3}} d r d \varphi+\frac{1}{\rho_{3}}\right] ; \\
\left.\frac{\partial \Phi}{\partial r}=-a^{2} \& \int_{0}^{L} \int_{0}^{2 \pi} \frac{R^{2}}{\left(\rho_{1}+\rho_{2}\right)^{2} \rho_{1}^{3}} d \varphi d z+\int_{0}^{R} \int_{0}^{2 \pi} \frac{r \cdot z_{C}}{\left(\rho_{1}+\rho_{2}\right)^{2} \rho_{1}^{3}} d r d \varphi+\frac{1}{\rho_{3}^{2}}\right]-\frac{1}{2} U a^{3}\left[\frac{\cos \theta_{1}}{\rho_{3}^{2}}+\frac{\cos \theta_{2}}{\rho_{4}^{2}}\right] ; \\
z_{C}=z_{C}(0)+\int_{0}^{t} U d t .
\end{gathered}
$$

Данные об изменении $a(t), \&(t)$, , необходимые для проведения расчета по формуле (1), при известном законе изменения давления в газовой полости $P_{\partial}(t)$ могут быть получены при решении уравнения Релея[1], описывающего процесс развития одиночной сферической полости в объеме невязкой, несжимаемой и лишенной капиллярных свойств жидкости:

$$
\&^{2}+\frac{3}{2} \& Z=\frac{P_{\partial}(t)}{\rho}-F(0,0,0, t) .
$$

В правой части уравнения Релея учитывается действие источников, расположенных на боковой поверхности цилиндрической емкости. В рассматриваемом случае уравнение перепишем как 


$$
\begin{aligned}
& 2+\frac{3}{2} \&^{2}=\frac{P_{\partial}(t)}{\rho}-\frac{P_{c m}}{\rho}-\left(2 a \&+a^{2} \int_{0}^{L} \int_{0}^{L 2 \pi} \frac{R^{2}}{\left(\rho_{1}+\rho_{2}\right) \rho_{1}^{3}} d z d \varphi+\int_{0}^{R} \int_{0}^{2 \pi} \frac{r \cdot z_{C}}{\left(\rho_{1}+\rho_{2}\right) \rho_{1}^{3}}\right]+ \\
& +\frac{1}{2}\left[-a^{2} \& \int_{0}^{L} \int_{0}^{2 \pi} \frac{R^{2}}{\left(\rho_{1}+\rho_{2}\right)^{2} \rho_{1}^{3}} d z d \varphi-a^{2} \& \int_{0}^{R} \int_{0}^{2 \pi} \frac{r \cdot z_{C}}{\left(\rho_{1}+\rho_{2}\right)^{2} \rho_{1}^{3}} d r d \varphi+\frac{U a^{3}}{8 z_{C}^{2}}\right]
\end{aligned}
$$

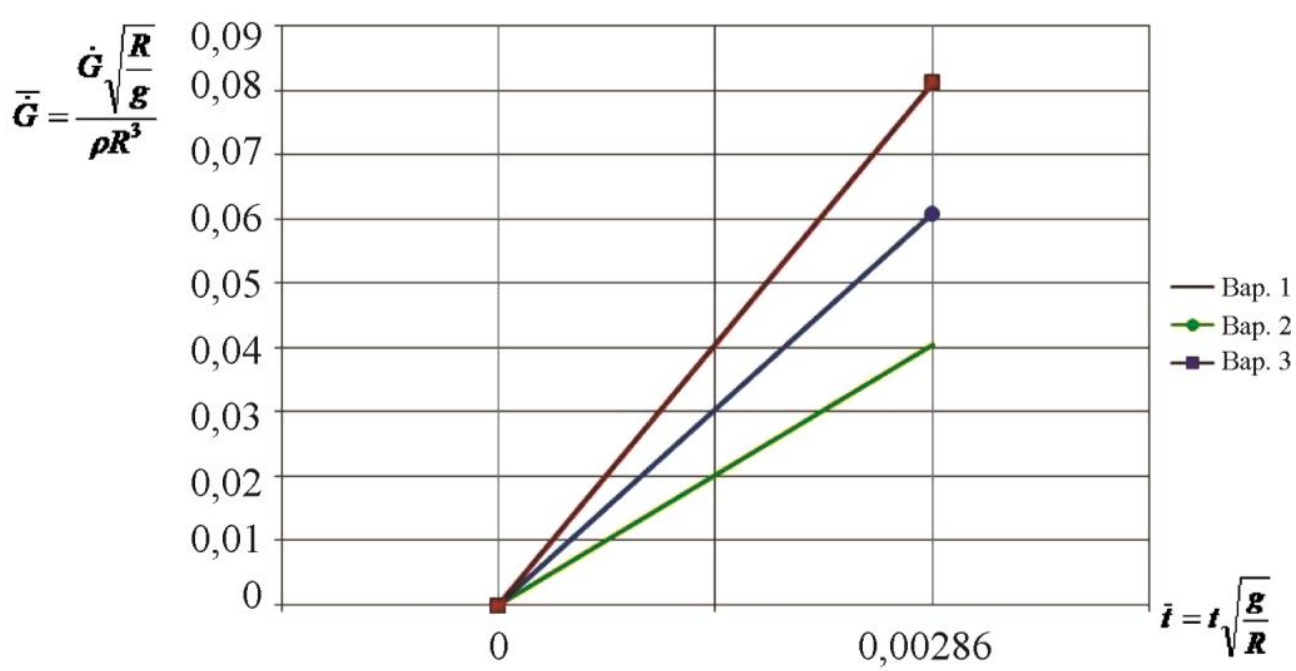

Рис. 3. Расход газа, поступающего в полость

Давление в газовой полости $P_{\partial}(t)$ определим из уравнения состояния идеального газа: $P_{\partial}(t)=\frac{3 m R_{2} T}{4 \pi a^{3}}$, где $R_{2}-$ газовая постоянная; $T-$ температура газа в полости; $m(t)=m_{0}+\int_{0}^{t} G d t-$ масса газа в полости; $\mathrm{G}-$ расход поступающего в полость газа.

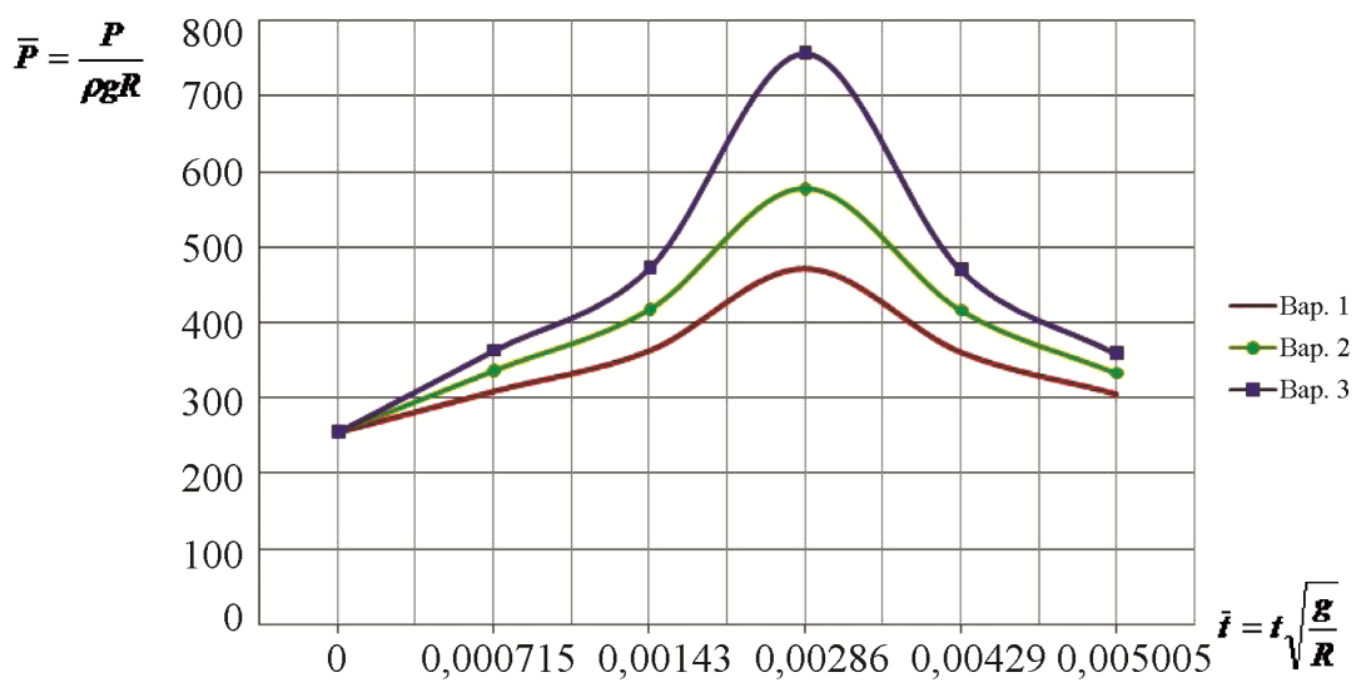

Рис. 4. Давление на стенки установки в ближайшей к газовой сфере точке 


\section{3. Результаты}

Расчет проведем для цилиндрической емкости радиусом 1,2 м (принятым за характерный линейный размер), заполненной водой на 4 м. На рис. 3 приведены графики расходов газа для трех расчетных случаев. Графики давления в ближайшей к источнику точке, расположенной на стенке экспериментальной установки, представлены на рис. 4.

\section{4. Заключение}

Приведенная методика расчета дает возможность определить поле давлений при интенсивном развитии находящейся под водой газовой полости и нагрузки на экспериментальную установку.

\section{Литература}

1. Кочин Н. Е., Кибель И. А., Розе Н. В. Теоретическая гидромеханика. - Москва : Физматгиз, 1963. - Т.1. - 584 с.

2. Милн-Томпсон Л. М. Теоретическая гидродинамика. - Москва : Мир, 1964. - 660 с.

3. Седов Л. И. Механика сплошной среды. - Москва : Наука, 1970. - Т. 2. - 568 с.

4. Чижиумов С. Д. Основы гидродинамики : учеб. пособие. - Комсомольск-на-Амуре : КнАГТУ, 2007. - 106 с.

5. Дегтярь В. Г., Пегов В. И. Гидродинамика баллистических ракет подводных лодок. Миасс : КБ им. акад. В. П. Макеева, 2004. - 256 с.

6. Дегтярь В. Г., Пегов В. И., Меркулов Е. С. Численное моделирование эволюции границы каверны при пуске торпеды // Вестник ЮУрГУ. Серия «Математическое моделирование и программирование». - 6 (1). - 2013 - С. 5-12.

7. Спевак Л. Ф., Нефедова О. А. Распараллеливание решения нелинейной задачи теплопроводности с использованием библиотеки OPENCL. - URL: http://dreamjournal.org/issues/2016-6/2016-6_113.html (дата обращения: 23.12.2021).

8. Kazakov A. L., Spevak L. F., Nefedova O. A. Simultenious Application of the Boundary Element Method and the Power Series Method for Solving a Two-Dimensional Problem of Heat Wave Motion // Diagnostics, Resource and Mechanics of materials and structures. 2017. - Iss. 6. - P. 6-15. - DOI: 10.17804/2410-9908.2017.6.006-015. - URL: http://dreamjournal.org/issues/2017-6/2017-6_151.html (accessed: 12.12.2018).

9. Pegov V. I., Moshkin I. Yu. Applying the Method of Plane Sections for Evaluating the Parameters of Flight Vehicles under Multiphase Flow // Diagnostics, Resource and Mechanics of materials and structures. - 2020. - Iss. 4 - P. 48-61. - DOI: 10.17804/2410-9908.2020.4.048-061. URL: http://dream-journal.org/issues/2020-4/2020-4_299.html (accessed: 23.12.2021).

10. Pegov V. I., Moshkin I. Yu. Applying an Updated Method of Markers to Defining Transient Force Impact under Multiphase Flowing // Diagnostics, Resource and Mechanics of materials and structures. - 2020. - Iss. 5. - P. 45-57. - DOI: 10.17804/2410-9908.2020.5.045-057. URL: http://dream-journal.org/issues/2020-5/2020-5_300.html (accessed: 23.12.2021). 